\title{
Objection de conscience: déontologie et service à la population
}

Les codes déontologiques prévoient que le médecin peut s'abstenir de fournir des prestations qui lui posent un problème de conscience. Ainsi pour l'interruption de grossesse (IG), pour la prescription de contraceptifs (y compris stérilisation), pour la procréation médicalement assistée (PMA). Comme la plupart, je suis attaché à ce principe.

Cependant, quand comme médecin cantonal j'étais consulté, je rappelais que refuser de donner des soins n'est déontologiquement licite qu'à plusieurs conditions:

- qu'il ne s'agisse pas d'une situation d'urgence où l'absence de traitement, ou un retard à le donner, a des conséquences négatives notables pour le/la patient(e);

- que ce refus n'entraîne pas pour la personne des difficultés sérieuses à obtenir la prestation, par exemple à cause de distances à parcourir, de conséquences matérielles et financières importantes ...;

- de plus, le médecin a le devoir déontologique, même s'il réprouve l'intention du patient, de l'informer adéquatement sur des confrères susceptibles de fournir la prestation.

Où la situation deviendrait problématique - prenant l'exemple de la gynécologie -, c'est l'éventualité que dans une région géographiquement étendue l'ensemble des praticiens allèguent l'objection de conscience. Alors, l'appréciation change: ce qui est admissible par respect des convictions de l'individu ne l'est plus, du point de vue de la société, si tous les prestataires adoptent la même position. En effet, cela entraînerait pour les patientes des obstacles majeurs à bénéficier près de leur domicile de prestations que la loi dit licites (dans le cadre nouveau depuis 2002, pour ce qui concerne l'IG, du régime du délai). Il n'est pas acceptable que cet accès soit empêché ou rendu très malaisé: question d'éthique civique, d'égalité devant la loi et les soins. La responsabilité des pouvoirs publics, particulièrement de l'autorité sanitaire (Conseiller d'Etat, médecin cantonal), serait alors engagée; cette autorité doit faire en sorte, d'une manière ou de l'autre, que les femmes de la région disposent des services en cause.

Une motion récente au Grand Conseil vaudois demande que le droit à l'objection soit ga- ranti légalement. Sur certains sujets cela a été une avancée, ces vingt dernières années, de promulguer des lois - ainsi sur les relations entre soigné et soignant, les droits des patients. Toutefois, les codes déontologiques gardent leur importance et ils reconnaissent l'objection de conscience. On doit donc d'abord se demander si le besoin d'une loi est avéré, en se souvenant que les actes concrets des professionnels ne sont en aucune manière réglés de façon exhaustive par la loi: vis-à-vis des gestes de soins, des dispositions impératives détaillées peuvent devenir des carcans. A mon sens, ce besoin n'est pas démontré; et une loi devrait tenir compte des exceptions cidessus, d'où un texte forcément compliqué aux effets discutables, pour traiter ce qui aujourd'hui est dans la pratique un non-problème (durant tout mon mandat officiel, nous n'avons pas vu de litiges à ce sujet).

Se posent aussi des questions de responsabilité d'une institution et de droit du travail: quelle attitude des responsables d'un hôpital public si, par hypothèse, une partie importante du personnel soignant refuse de participer à certaines interventions? Quand un(e) employé(e) parmi vingt ou trente a ce souci de conscience, sans doute cela peut-il être géré dans le dialogue; mais si dix ou quinze ont la même position ... Qu'estce alors qui prime? Pour le moins, on ne saurait négliger le besoin que l'établissement remplisse sa mission au service des patient(e)s. On se souvient que, après l'adoption du régime du délai, un hôpital de Suisse centrale s'est séparé de son gynécologue-chef parce que ce dernier refusait la pratique d'IG dans le service.

Je soutiens le principe déontologique de l'objection de conscience mais la démarche parlementaire vaudoise évoquée illustre la réflexion à mener avant de requérir que l'Etat intervienne dans la vie des professions et des citoyens. En conclusion: respect de la conscience du médecin, oui sans doute. Mais garder à l'esprit que la responsabilité qu'il a accepté d'assumer vis-à-vis de son institution et de la population peut légitimement mettre des limites au droit à l'objection. Etant entendu aussi que le praticien peut être attentif à ne pas accepter de mandats où il se trouverait ainsi contraint.

Jean Martin* daction, est ancien médecin cantonal vaudois et membre de la Commission nationale d'éthique. 